CARNETS DE Carnets de géographes

GÉOGRAPHES.

$7 \mid 2014$

Les espaces de l'entre-deux

\title{
Géopolitique de la Chine en Cote d'Ivoire
}

La puissance chinoise à l'école ivoirienne et africaine

Xavier Aurégan

(2) OpenEdition

Journals

Édition électronique

URL : http://journals.openedition.org/cdg/394

DOI : $10.4000 /$ cdg.394

ISSN : 2107-7266

Éditeur

UMR 245 - CESSMA

Référence électronique

Xavier Aurégan, « Géopolitique de la Chine en Cote d'Ivoire », Carnets de géographes [En ligne], 7| 2014, mis en ligne le 01 décembre 2014, consulté le 23 septembre 2020. URL : http://

journals.openedition.org/cdg/394; DOI : https://doi.org/10.4000/cdg.394

\section{(c) (i) $\odot$}

La revue Carnets de géographes est mise à disposition selon les termes de la Licence Creative Commons Attribution - Pas d'Utilisation Commerciale - Pas de Modification 4.0 International. 


\section{GEOPOLITIQUE DE LA CHINE EN COTE D'IVOIRE}

\section{La puissance chinoise à l'école ivoirienne et africaine}

\section{XAVIER AURÉGAN}

Cette recherche a pour ambition l'analyse des relations sino-africaines par le prisme ivoirien. S'appuyant sur six études de terrain effectuées dans le cadre du Master Recherche de Géopolitique (Institut français de géopolitique - université Paris VIII) et durant le Doctorat, cette thèse embrasse l'ensemble des enjeux induits par ce nouveau paradigme de la géopolitique africaine. La présence chinoise, en Afrique subsaharienne et notamment en Afrique de l'Ouest francophone, doit être conjuguée au pluriel, la pluralité des présences étant l'une des principales spécificités du phénomène global dénommé Chine-Afrique.

Résolument transdisciplinaire et essentiellement empirique, cette recherche aborde l'ensemble des thématiques ou facteurs considérés comme essentiels à la compréhension multiscalaire de la relation sino-africaine, et ainsi sino-ivoirienne. Ces derniers sont liés aux migrations et aux acteurs chinois, à l'aide au développement chinoise - et la coopération afférente -, à la place du partenaire ivoirien à l'échelle de la Chine-Afrique, et enfin à l'implantation multimodale de la "Chine» sur un territoire et au sein d'un État «sortant» de plusieurs crises structurelles.

La première partie analyse l'ensemble des présences chinoises en Côte d'Ivoire, ce faisceau diversifié d'acteurs et d'activités, soit les acteurs humains (chinois) implantés sur le territoire ivoirien, abidjanais notamment, puis ces acteurs indirects de la coopération chinoise. Il s'agit principalement de commerçants, de personnels de santé, d'hôteliers, de gérants de salons de massages, d'entrepreneurs et de fonctionnaires, de techniciens et de personnels liés au gouvernement chinois... mais également des disparates productions de savoirs ou des coopérations décentralisées (jumelages). Ayant comme support une cartographie par communes, par quartiers voire, par rues, la localisation de ces acteurs, leurs origines géographiques et leurs activités sont ainsi étudiées par le biais de multiples entretiens et d'un questionnaire sino-français. Les représentations dont ils sont victimes, qu'ils créent, entretiennent et diffusent au sein de leurs " communautés économiques » et de la population ivoirienne et ouest-africaine sont intégrées à l'analyse des conflits, des rivalités de pouvoirs et des enjeux liés aux conditions de départs (Chine) et d'arrivée (Côte d'Ivoire). C'est l'envers du décor des huáqiáo (ressortissants chinois) à Abidjan, où intégration, structure migratoire et rôles des États se mêlent.

Après avoir entrepris cette analyse globale via les géographies humaine et sociale, voire par l'anthropologie, la deuxième partie se consacre, quant à elle, à la coopération sino-ivoirienne et aux domaines d'interventions économiques, des "médecins aux pieds nus » et des agronomes jusqu'aux nouveaux secteurs (télécommunications par exemple). L'approche géoéconomique permet effectivement d'analyser au mieux ces relations sino-africaines, par nature économiques. Ce primat de l'économie est prégnant depuis le milieu des années 1990 et le lancement de plusieurs politiques chinoises assurant, à Beijing, les moyens de ses investissements, des échanges commerciaux et des coopérations multilatérales. Les acteurs 
économiques chinois, les « cas particuliers »-joint venture - sont incorporés dans cette analyse économique où la cartographie tient toujours une place prépondérante. La Chine, en tant qu'acteur étatique et représenté comme tel - contrairement à la première partie -, est devenue le principal partenaire du continent africain en 2010. Une " présence » et une influence économique extrêmement disparates, en partie dues à la situation endogène des États africains et donc des principales puissances régionales. Cette partie " déconstruit » par ailleurs l'aide publique au développement chinoise, du Congrès national du Peuple à l'ambassade africaine, et en étudie les principaux moyens et effets. Dans un souci d'exhaustivité et d'objectivité, celle-ci est comparée à l'aide européenne et à celle de l'autre «Chine » historiquement implantée en Côte d'Ivoire: Taïwan (1960-1983). Ces mises en perspective effectuent une transition vers la troisième partie, géohistorique et déplacent le curseur au niveau du continent africain.

L'avant-dernière partie intègre ainsi « l'objet géographique » qu'est la Côte d'Ivoire et "l'objet géopolitique » qu'est la relation sino-ivoirienne dans le partenariat global ChineAfrique, depuis les indépendances africaines jusqu'au dernier sommet sino-africain de 2012. Les étapes majeures de ce rapport multilatéral (mais in fine bilatéral) sont analysées, de la proclamation de la RPC, en 1949, en passant par la Conférence de Bandung (1955), l'accession de Beijing au CSNU (1971), aux réformes endogènes (1971) et jusqu'aux réformes touchant l'APD chinoise (1994 puis 2000 avec le premier sommet Chine-Afrique). Depuis la fin des années 1950, la Chine populaire se trouve dans le(s) jeu(x) économique(s) et politique(s) africain(s), et l'économie, comme la géopolitique, sont au service des partenariats multi et bilatéraux.

La dernière partie est finalement consacrée aux deux principaux prismes analytiques, la géographie économique et l'analyse géopolitique. En croisant ces deux facteurs, il est incontestable que la «crise » a accéléré le «bond» économique et commercial chinois en Côte d'Ivoire. Dans cet État ayant connu plusieurs crises successives (1995, 1999, 2002 et 2010-2011), quel est le rôle de cette puissance émergente, sa position lors du conflit postélectoral de 2010-2011, quels sont les discours de Pékin comme de ses représentants locaux, et que disent les cartographies des déplacements du personnel de l'ambassade ou des visites officielles? Ces quelques interrogations trouvent ainsi leurs réponses à travers les quatre temps de la relation sino-ivoirienne (1983-1993; 1994-1999 ; 2000-2010 ; 2011-2013), qui représentent, en définitive, les quatre présidents ivoiriens depuis la reconnaissance entre la Côte d'Ivoire et la puissance chinoise, pragmatique et forgeant son expérience africaine à travers le cas ivoirien. 
Fiche informative

\section{Discipline}

Géographie-géopolitique

\section{Directeurs}

Alain Gascon et Alphonse Yapi-Diahou

\section{Université}

Institut français de géopolitique-université Paris 8

Membres du jury de thèse, soutenue le 04/03/2014

- Thierry Pairault (président)

- Céline Bikpo (rapporteur)

- Jean-Jacques Gabas (rapporteur)

- Jean-Fabien Steck (membre du jury)

- Alain Gascon (codirecteur)

- Alphonse Yapi-Diahou (codirecteur)

\section{Situation professionnelle actuelle}

Chargé d'enseignement aux universités Paris 1 et Paris 10 - Chercheur associé au CIRAD

\section{Courriel de l'auteur}

xavierauregan@hotmail.com 